\title{
Combination of Neutral Zone and Flange Techniques during Fabrication of Complete Dentures for a Segmental Mandibulectomy Patient with Restricted Mouth Opening
}

\author{
${ }^{1}$ Rahul S Kulkarni, ${ }^{2}$ Ravindra S Pawar
}

\begin{abstract}
Oral malignancies originating from or secondarily involving mandible are usually treated by surgical excision and may result in mandibular defect with or without loss of continuity. Surgical resection may lead to loss of buccal and lingual sulci, loss of symmetry of mandibular function, altered mandibular movements and deviation of the residual fragment toward the resected side. Radiation therapy is often given after resection of malignant tumors, which may produce undesirable side effects, like mucositis and mucosal atrophy, trismus, increased susceptibility to irritation and ulceration and reduction in salivary flow. This overall change in anatomy and physiology of oral cavity after segmental mandibular resection presents a restorative challange. This clinical report presents fabrication of complete dentures for a patient who had undergone segmental mandibulectomy and had restricted mouth opening.
\end{abstract}

Keywords: Complete dentures, Flange technique, Mandibulectomy, Neutral zone.

How to cite this article: Kulkarni RS, Pawar RS. Combination of Neutral Zone and Flange Techniques during Fabrication of Complete Dentures for a Segmental Mandibulectomy Patient with Restricted Mouth Opening. Int J Prosthodont Restor Dent 2015;5(4):114-117.

\section{Source of support: Nil \\ Conflict of interest: None}

\section{INTRODUCTION}

Oral malignancies, like carcinomas originating from floor of mouth, lateral border of tongue, or buccal mucosa may secondarily involve mandible, and ablative surgeries directed at removal of these tumors may lead to resection of a part of the mandible. Mandibular defects may be broadly classified into three types as marginal and segmental mandibulectomy, and hemimandibulectomy, depending on the amount of bone resection carried out. ${ }^{1}$ Surgical resection may lead to loss of continuity of the mandible,

${ }^{1}$ Associate Professor, ${ }^{2}$ Assistant Professor

1,2Department of Prosthodontics, Nair Hospital Dental College Mumbai, Maharashtra, India

Corresponding Author: Rahul S Kulkarni, Associate Professor Department of Prosthodontics, Nair Hospital Dental College Mumbai, Maharashtra, India, Phone: 02223082714, e-mail: drrahulprostho@yahoo.com loss of symmetry of mandibular function, altered mandibular movements and deviation of the residual fragment toward the resected side. Loss of soft and hard tissues from surgery and contraction during healing and radiotherapy may result in restriction of mouth opening. ${ }^{2}$ Surgical procedures are usually combined with microvascular reconstruction to reduce morbidity and improve the prognosis of rehabilitation. Radiation therapy is often given after resection of malignant tumors, which may produce undesirable side effects like, mucositis and mucosal atrophy, trismus, increased susceptibility to irritation and ulceration, and reduction in salivary flow. The magnitude of postsurgical disability is often related to the structures involved and the extent of resection, and reduction in basal support foundation of mandibular dentures may reduce its retention and stability. ${ }^{3}$ Stability of mandibular complete denture is further compromised due to loss of the normal integrated neuromuscular control resulting from tissue loss. This overall change in anatomy and physiology of oral cavity after segmental mandibular resection presents a restorative challenge. This clinical report presents fabrication of complete dentures for a patient who had undergone segmental mandibulectomy and had restricted mouth opening.

\section{CLINICAL REPORT}

A 60 years old completely edentulous female presented for treatment seeking replacement of her missing teeth. The patient was diagnosed with squamous cell carcinoma of right buccal mucosa in 2010 and was treated with wide excision, segmental mandibulectomy and reconstruction using a left ulnar free flap, followed by radiotherapy. Patient had restricted mouth opening and there was a deviation of the mandible to the resected side (Fig. 1). Intraoral examination revealed completely edentulous arches and complete obliteration of maxillary and mandibular buccal and lingual vestibules on right-side resulting from tumor resection. Further examination revealed loss of alveolar ridge on right side (Fig. 2), restricted tongue movements, and normal anatomy of supporting structures on left side. Reconstructive plastic surgery was indicated to create buccal and lingual sulcus on right side to improve prognosis of mandibular denture, 


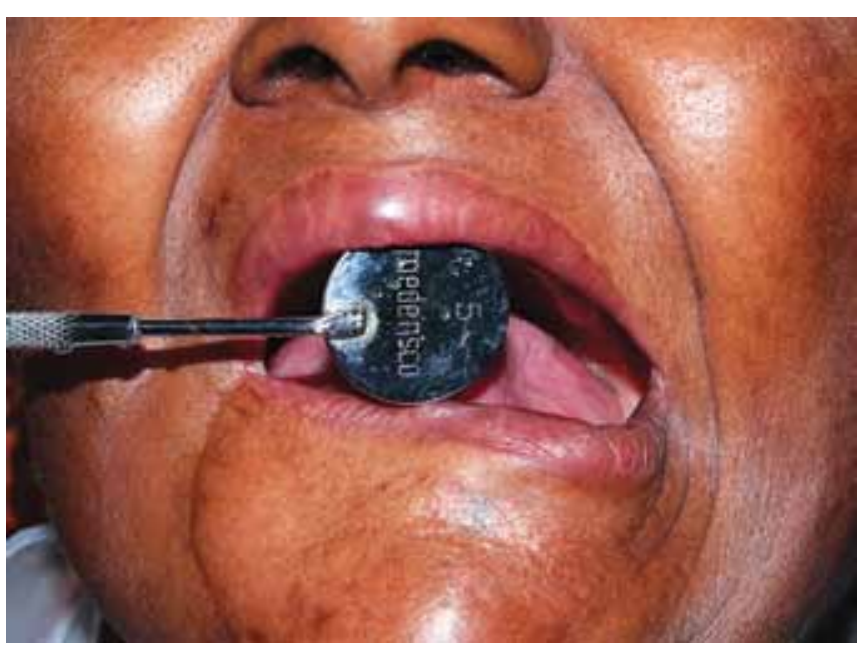

Fig. 1: Extraoral view of patient. Note maximum mouth opening is approximately of diameter of an intraoral mirror

however, patient declined the treatment option due to fear of surgery. Patient did not accept implant supported overdentures due to financial constraints and consented for conventional complete dentures (CCD).

Maxillary and mandibular preliminary impressions were made with medium fusing impression compound (Y-dents, MDM Corporation, New Delhi, India) in modified stock edentulous trays. The stock trays were modified by trimming on dental lathe to conform to the residual ridge contours on the resection side. Primary casts were obtained from the impressions, and custom trays were fabricated using autopolymerizing PMMA resin (Acrylin, DPI Ltd., Mumbai, India). Maximum coverage of denture foundation tissues within their physiologic limits of tolerance was planned during tray fabrication to maximize the retention and support. Border molding was performed in incremental manner using low fusing modeling plastic (DPI Pinnacle, DPI Ltd., Mumbai, India), and final impressions were made using medium body polyvinylsiloxane impression material (Honigum, DMG, Hamburg, Germany) (Fig. 3). During border

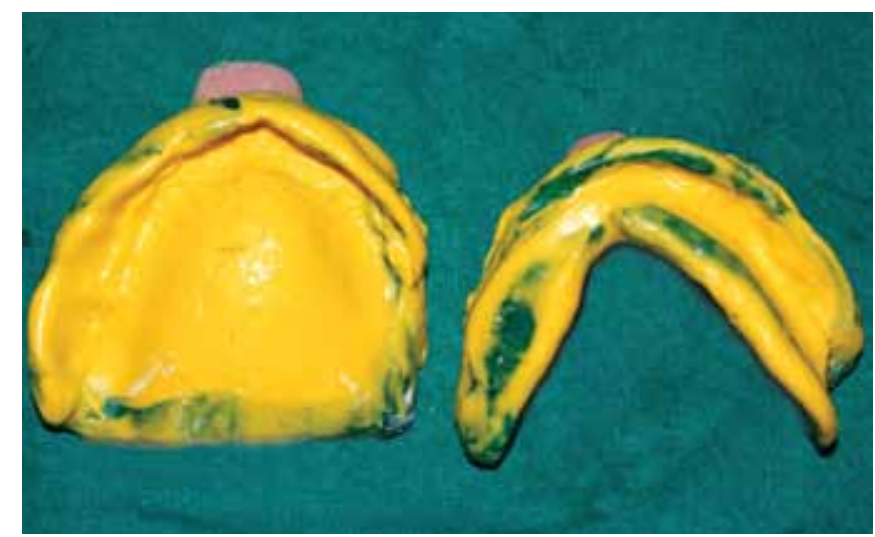

Fig. 3: Maxillary and mandibular final impressions slight overextension in anterior lingual sulcus and sublingual crescent region (on normal side) is usually well tolerated and adds remarkably to denture retention

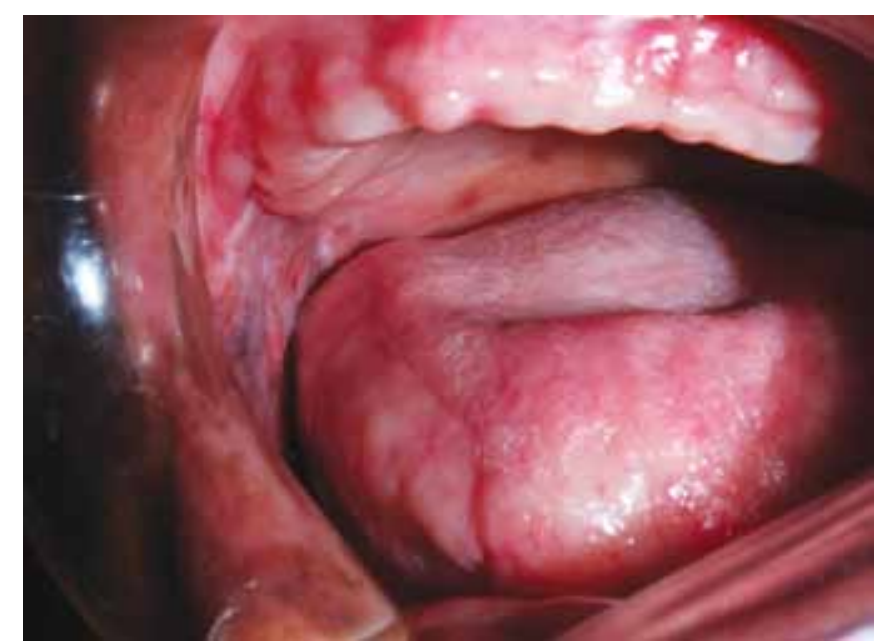

Fig. 2: Intraoral view exhibits total absence of buccal and lingual sulcus in mandibular arch and obliteration of maxillary buccal vestibule

molding of mandibular special tray, careful extension of the modeling plastic was done in anterior lingual region and sublingual crescent region to maximize retention. ${ }^{4}$ Record bases and wax rims were fabricated on working casts using shellac base plates (Maarc, Shiva Products, Thane, India) and baseplate wax (Modeling wax, Shiva products, Thane, India) respectively. Necessary modifications were done in wax rims to accommodate for altered anatomy of residual ridges on right side. Face bow transfer, vertical jaw relations and centric relations were recorded using standard techniques. After completion of jaw relation records and mounting on semiadjustable articulator, width of mandibular wax rim was reduced to half, and neutral zone was recorded using resilient liner (Soft-Liner, GC Corp, Tokyo, Japan) (Fig. 4). ${ }^{5}$ Silicone putty index was fabricated from the neutral zone record (Coltene Rapid Base, Knetmasse Putty Material; Coltene/ Whaledent Inc, Mahwah, NJ), and teeth arrangement was done using semianatomic teeth (IvoStar, Ivoclar Vivadent, Schaan, Liechtenstein) (Fig. 5). ${ }^{6}$ A second row of teeth in

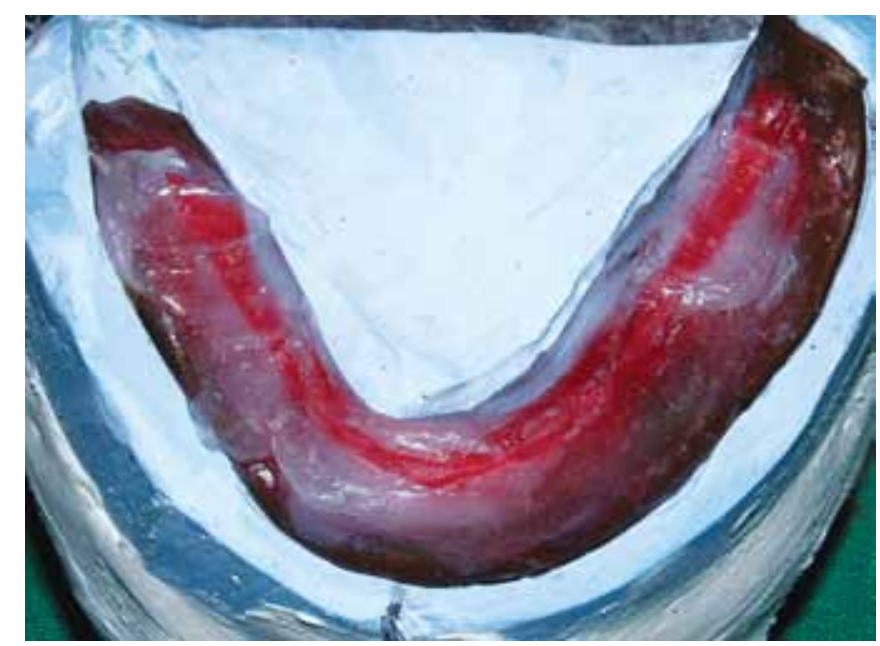

Fig. 4: Neutral zone record in mandibular arch 
maxillary arch was deemed unnecessary since patient could easily close in centric relation and only marginal discrepancy was noted in overlap of arches at closure. The second mandibular molar was eliminated on the resection side to limit the loading on that side. Try-in of waxed up dentures was carried out to evaluate function and esthetics in the conventional manner. At the completion of try in, application of 'flange technique' was carried out on mandibular denture as per the following procedure. ${ }^{7}$

\section{PROCEDURE}

- Place maxillary trial denture in patients mouth. If retention of denture is inadequate, application of denture adhesive is recommended.

- Remove wax from buccal, labial and lingual surfaces of mandibular trial denture to make space for resilient liner. Add sufficient amount of resilient liner (SoftLiner, GC Corporation, Tokyo, Japan) on buccal surface of the denture, to slightly exceed in amount than initial waxing and carving. Insert trial denture in patient's mouth before curing of the liner, so that molding of liner by tissues can be carried out.

- Ask the patient to swallow, grin, pucker and purse the lips, speak aloud and make other movements of lip till curing of liner takes place. Remove mandibular denture, make additions of liner in deficient areas, and again repeat molding to complete the procedure on buccal and labial sides (Fig. 6).

- Apply liner on lingual side of the trial denture, place denture in mouth and secure it in place with digital pressure, and ask patient to perform various tongue movements like protrusion, gentle licking of upper lip, touching each cheek and swallowing. Continue the movements till material sets, and repeat the procedure if required (Fig. 6).

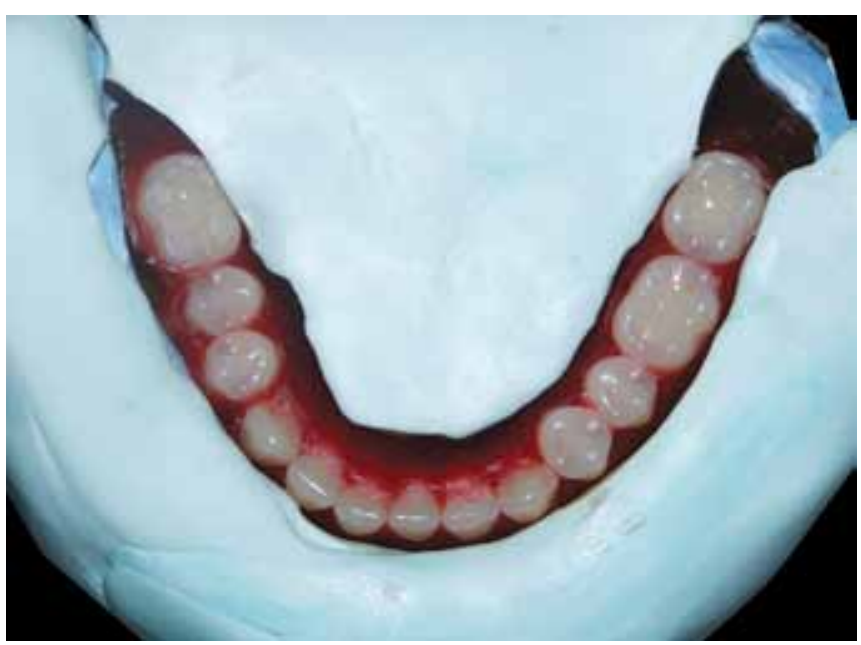

Fig. 5: Tooth arrangement done following index made from the neutral zone record

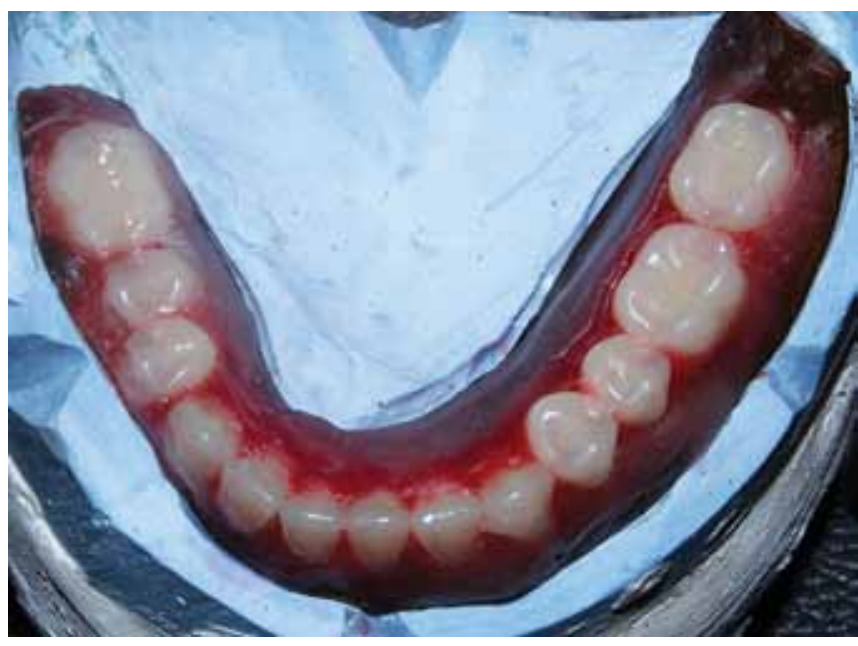

Fig. 6: Modification of mandibular trial denture by 'flange technique' using resilient liner. The liner seen has been molded by movements of tissues surrounding denture

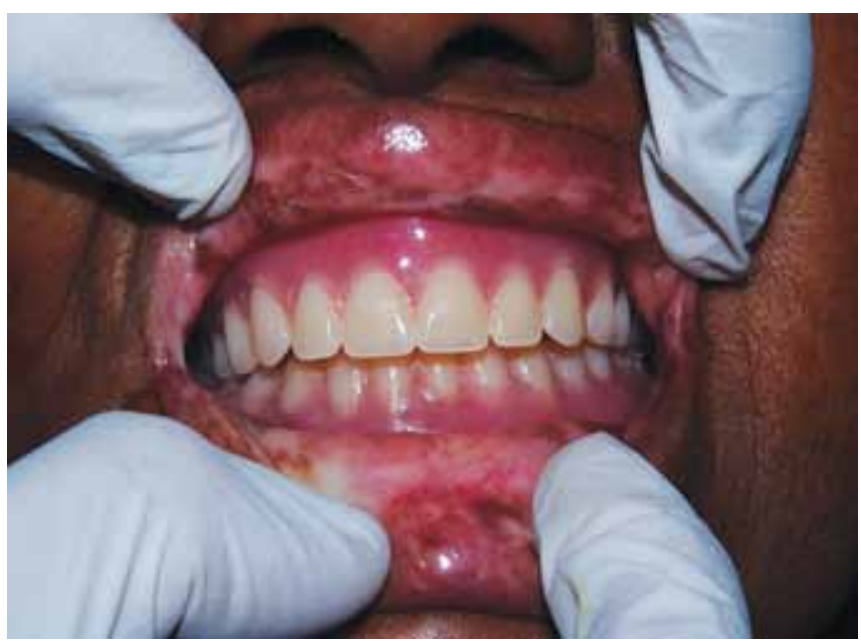

Fig. 7: Intraoral view of complete dentures after processing

- Retrieve the trial dentures and carry out the processing, and perform laboratory remount and finishing and polishing of dentures.

- At the denture insertion appointment, instruct the patient regarding in the insertion and removal of the dentures, oral and denture hygiene procedures, and schedule periodic recalls at regular intervals (Fig. 7).

\section{DISCUSSION}

A patient who has undergone tumor ablative surgery and radiotherapy may present with several alterations in stomatognathic system, like reduction in basal seat area and salivary flow, atrophy of the oral mucosa, deviation of mandible, reduction in neuromuscular control, and restriction of mouth opening. ${ }^{8,9}$ To overcome these limitations, principles of denture construction must be applied meticulously to obtain maximum retention, support, stability, esthetics and function. Retention and stability in dentures depends on three surfaces viz impression surface, occlusal surface and polished denture 
surface. While adequate attention is usually given to the impression procedures and development of desirable occlusion in dentures, the anatomy of polished surface is often neglected. ${ }^{7}$

The concept of 'Neutral Zone' was first propounded by Fish in 1933, and the term neutral zone describes the area or position where forces between the tongue and cheeks or lips are equal. ${ }^{5}$ The neutral zone concept was applied during treatment of this patient to enhance the stability of the mandibular denture. Lott and Levin observed that polished surfaces of denture are usually carved into an 'ideal' form without sufficient consideration for the position and function of cheeks, tongue and lips with which it is constantly in close contact and with which it must function in harmony. For instance, buccinator remains in close contact with buccal surfaces of molars and gingivae in natural dentition, however, a space exists between buccinator and anatomically carved denture flanges. ${ }^{7}$ They described the 'flange technique' to develop denture surface that is intimately adapted to the cheeks, tongue and lips and that will function in harmony with these tissues. The technique is simple, rapid, easy to apply, and leads to improvement in retention and masticatory efficiency. Hence the 'flange technique' was combined with the neutral zone technique to adequately record relation of polished denture surfaces to oral tissues. Since the mandibulectomy patients have reduction in alveolar support, it is imperative to record and utilize maximum denture base area possible within the physiological limits. Hence, particular attention was given to record depth of anterior lingual sulcus and sublingual crescent region for optimum retention. ${ }^{4}$

Prosthodontic literature contains reports of mandibulectomy patients treated with CCD and sectional or collapsible dentures. ${ }^{2,3,6}$ A notable complaint observed in mandibulectomy patients with CCD has been 'ingress of food beneath the denture', which is later corrected by soft or hard reliner. Ohkubo has advocated use of neutral zone technique for tooth arrangement and relining the polished surface of denture chairside with hard liner, a technique he termed as 'border molding' ${ }^{3}$ In these reports, polished surface of the denture has been modified after processing, with inherent limitations related to use of soft and hard reliners. In the present case, movements of lips, cheeks and tongue were recorded in trial dentures using resilient liners before dentures were processed, requiring no alterations of the polished surface post insertion. A sectional denture could be fabricated in present case, however, it has disadvantages like complicated processing technique, potential for repair and maintenance and sometimes tissue abuse like pinching. The complete dentures fabricated for this patient by combination of neutral zone and flange techniques served adequately for purpose of mastication and esthetics, and no post insertion problems were reported.

\section{SUMMARY}

This article presents information on prosthodontic management of a patient treated with segmental mandibulectomy and having restricted mouth opening. Bone grafting followed by implant therapy would be the ideal treatment, however, complete dentures were fabricated for the patient since, she was not willing to undergo surgery and implant therapy. The denture prostheses restored speech, mastication, and esthetics and enhanced overall well being of the patient.

\section{REFERENCES}

1. Kadota C, Sumita YI, Wang Y, Otomaru T, Mukohyama H. Comparison of food mixing ability among mandibulectomy patients. J Oral Rehabil 2008;35(6):408-414.

2. Suzuki Y, Abe M, Hosoi T, Kurtz KS. Sectional collapsed denture for a partially edentulous patient with microstomia: a clinical report. J Prosthet Dent 2000;84(3):256-259.

3. Ohkubo C, Hanatani S, Hosoi T, Mizuno Y. Neutral zone approach for denture fabrication for a partial glossectomy patient: a clinical report. J Prosthet Dent 2000;84(4):390-393.

4. Chang JJ, Chen JH, Lee HE, Chang HP. Maximizing mandibular denture retention in the sublingual space. Int $\mathrm{J}$ Prosthodont 2011;24(5):460-464

5. Wee AG, Cwynar RB, Cheng AC. Utilization of the neutral zone technique for a maxillofacial patient. J Prosthodont 2000; 9(1):2-7.

6. Mou SH, Chai T, Shiau YY, Wang JS. Fabrication of conventional complete dentures for a left segmental mandibulectomy patient: a clinical report. J Prosthet Dent 2001;86(6):582-855.

7. Lott F, Levin B. Flange technique: an anatomic and physiologic approach to increased retention, function, comfort and appearance of dentures. J Prosthet Dent 1966;16(3):394-413.

8. Watanabe I, Tanaka Y, Ohkubo C, Miller AW. Application of cast magnetic attachments to sectional complete dentures for a patient with microstomia: a clinical report. J Prosthet Dent 2002;88(6):573-577.

9. Cheng AC, Wee AG, Morrison D, Maxymiw WG. Hinged mandibular removable complete denture for post-mandibulectomy patients. J Prosthet Dent 1999;82(1):103-106. 